\title{
Funcionamiento familiar y resiliencia en pacientes con enfermedades no transmisibles de un hospital público de Lima Metropolitana
}

\section{Family functioning and resilience in patients with noncommunicable diseases of a public hospital in Metropolitan Lima}

\author{
Moises Céspedes Felix ${ }^{1}$, Jesly Nina Cayte ${ }^{2}$, Wildman Vilca Quiro ${ }^{3}$
}

\section{RESUMEN}

Objetivo: estudiar la relación entre las variables funcionamiento familiar y resiliencia en pacientes con enfermedades no transmisibles de un hospital público de Lima. Material y métodos: de la investigación fue de diseño no experimental, de corte transversal y de alcance correlacional. El modelo del funcionamiento familiar sobre el cual se trabajó fue el modelo Circumplejo de sistema familiar realizado por Olson, Russell y Sprenkle (1979). La escala utilizada para la medición del funcionamiento familiar fue la Escala de Evaluación de Cohesión y Adaptabilidad Familiar (FACES III) y para medir la resiliencia se utilizó la Escala de Resiliencia elaborado por Wagnild y Young (1993) que consta de cinco dimensiones. La muestra estuvo conformada por 109 pacientes. Resultados: Se encontró que el funcionamiento familiar no se relaciona con la resiliencia $(x 2=5,972 ; p>.05)$. Conclusiones: que cualquiera sea el nivel de funcionamiento familiar, es posible que el paciente sea resiliente; ya que existen factores individuales que intervienen en el desarrollo de la resiliencia.

Palabras clave: Funcionamiento familiar, resiliencia, sentirse bien solo, ecuanimidad, perseverancia, satisfacción personal, confianza en sí mismo.

\section{ABSTRACT}

Objective: of the present investigation was to: study the relationship between family functioning and resilience variables in patients with noncommunicable diseases of a public hospital in Lima. Material and Methods: of the research was non-experimental, cross-sectional and correlational in scope. The model of family functioning on which we worked was the Circumplex family system model by Olson, Russell and Sprenkle (1979). The scale used to measure family functioning was the Family Cohesion and Adaptability Assessment Scale (FACES III) and to measure resilience, the Resilience Scale developed by Wagnild and Young (1993) was used, which consists of five dimensions. The sample consisted of 109 patients. In the Results it was found that family functioning is not related to resilience $(x 2=5.972, p>.05)$. Therefore, it is Conclussion: that whatever the level of family functioning, it is possible that the patient is resilient; since there are individual factors that intervene in the development of resilience.

Keywords: Family functioning, resilience, feel good alone, equanimity, perseverance, personal satisfaction and self-confidence. 


\section{INTRODUCCIÓN}

A lo largo del tiempo, las personas se han venido enfrentando a situaciones violentas o amenazantes, asimismo enfrentan eventos adversos consecutivamente de distintas maneras (Bonanno, 2004). Dentro del amplio repertorio de adversidades que cualquier persona pueda experimentar, una de ellas son las enfermedades crónicas o conocidas también como Enfermedades No Transmisibles (ENT). En este contexto, la Organización Mundial de la Salud (OMS, 2017) afirma que 40 millones de sujetos fallecen anualmente debido a las ENT, la cual representa al $70 \%$ de muertes producidas en el mundo y que a su vez están en aumento. Entre estas cifras, las enfermedades cardiovasculares representan a 17,7 millones de muertes, el cáncer 8,8 millones de muertes, las enfermedades respiratorias 3,9 millones de muertes y la diabetes 1,6 millones de muertes anuales (OMS, 2017).

Frente a estas enfermedades la familia es el principal apoyo material y sobretodo emocional durante el proceso médico, puesto que el acompañamiento, la comunicación, la búsqueda de soluciones y provisión de cuidados, son factores fundamentales para minimizar el impacto de la enfermedad (Ledón, 2011).

Olson (1979) define el funcionamiento familiar como la capacidad que tiene la familia para hacer frente a las dificultades, crisis y las interacciones interpersonales; se fundamenta en la libertad, autonomía, estima y el espacio individual entre los miembros del hogar, que se establece mediante la comunicación entre sus miembros. Además, Leyva-Jiménez, Hernández-Juárez, Nava-Jiménez y López-Gaona (2007) mencionan que el funcionamiento familiar es la disposición de la familia antes y durante los periodos vitales los cuales pueden estar acompañados de dificultades, sin embargo, logran salir satisfactoriamente de ellas. Florenzano (1998) añade que la familia es un sistema funcional cuando tiene la capacidad de poder cumplir sus funciones, afrontar y superar todos los periodos de vida de cada miembro y a su vez lograr superar toda crisis familiar que pueda sobrevenir. Asimismo, señala que es un factor que determina la preservación de la salud de los integrantes de la familia (Florenzano, 1995).

No cabe duda que las ENT pueden alcanzar y afectar a la mayor parte de personas. En esta dirección, Becoña (2006) refiere que la resiliencia es la aptitud que posee el individuo para atravesar satisfactoriamente circunstancias negativas, logrando un desarrollo óptimo y triunfante a pesar del contexto desfavorable en la que se pueda encontrar. Juárez (2012) añade que la resiliencia es una respuesta innata que cada persona manifiesta genuinamente frente a adversidades; ésta beneficia la vida de un paciente crónico, inicialmente para ayudarle a recuperarse de la primera impresión de la noticia, el diagnóstico de la enfermedad y posteriormente proporciona medios para canalizar de manera productiva su experiencia. De esta manera Wagnild y Young (1993) declaran que la resiliencia es un rasgo positivo de las personas que ayuda a disminuir las respuestas negativas del estrés para un mejor afronte, logrando soportar las dificultades y de esta forma actuar de forma correcta ante la adversidad. Asimismo, añaden que la resiliencia presenta cinco dimensiones que se interrelacionan.

Siendo: satisfacción personal, la capacidad para entenderelsignificado delaviday comolapersona aporta en esta; ecuanimidad, la autopercepción equilibrada de la vida y experiencias, además la capacidad para llevar las situaciones con adecuadamente y tranquilidad, moderando las actitudes frente a la adversidad; sentirse bien solo, el significado a la libertad, la sensación de ser únicos e importantes; confianza en sí mismo, la capacidad del ente para creer y confiar en sus capacidades, habilidades y aptitudes, además logra sacar provecho de sus debilidades; y perseverancia, la fuerza que ayuda a persistir frente al desaliento o adversidad, promueve el anhelo de logro y autodisciplina (Wagnild y Young, 1993).

Por ende, es necesario mencionar que Athié y Gallegos (2009) señalan que la resiliencia y el funcionamiento familiar se relacionan debido a que permite que las personas puedan desarrollar un sentido más optimista y esperanzador, de modo que se pueda lidiar efectivamente con las adversidades del futuro.

Por todo lo expuesto anteriormente, el objetivo de este estudio es comprobar la relación entre funcionamiento familiar y resiliencia en pacientes adolescentes con ENT en un hospital público de Lima Metropolitana. 


\section{MATERIALES Y MÉTODO}

Este estudio fue de enfoque cuantitativo y de diseño no experimental, debido a que las variables de estudio no fueron controladas ni manipuladas. Siendo de corte transversal, debido al proceso de recolección de la información, puesto que se dio en un único momento. Por último, esta investigación tuvo un alcance correlacional porque buscó la relación entre las variables de estudio (Hernández, Fernández y Baptista, 2005).

\section{Participantes}

La muestra se obtuvo a través de una selección no probabilística por conveniencia. Participaron 109 pacientes del servicio de psicología del Instituto de Salud Especializado III-2 "Guillermo Almenara Yrigoyen" diagnosticados con alguna enfermedad no transmisible.

Con relación a los datos sociodemográficos: el $50.5 \%$ de los pacientes fueron varones, la mayoría (85.3\%) tiene de 15 a 17 años de edad. Asimismo, la mayoría vivía con ambos padres (73.4\%). Por otro lado, se observa un $29.4 \%$ de los pacientes con diagnóstico de alguna enfermedad oncológica; cabe resaltar que hay un $26.6 \%$ con diagnóstico de otras ENT como epilepsia, insuficiencia renal, obesidad, entre otras. Asimismo, hay un grupo considerable (35.8\%) de pacientes con más de 6 años de enfermedad.

\section{Instrumentos}

Se utilizaron las siguientes escalas:

Escala de Evaluación de Cohesión y Adaptabilidad Familiar (FACES III)

EI FACES III fue creado por Olson, Portner y Lavee en 1985, además fue traducido y validado en Lima por Reusche (1994). Esta escala permite evaluar cómo ven actualmente las personas (lo percibido) y cómo les gustaría que fuera (lo ideal) su funcionamiento familiar, por medio de dos dimensiones que son la cohesión y adaptabilidad familiar. Consta de 20 ítems, 10 de cohesión y 10 de adaptabilidad con un tipo de respuesta Likert, donde Nunca es equivalente a 1 y Siempre es 5 . La Escala total presenta una buena consistencia interna $r=.68$. Respecto a la fiabilidad global de la escala, es de .79; en cuanto a cohesión es .72 y finalmente en adaptabilidad es .66 (Olson, Portner y Lavee, 1985).
Además, para esta investigación, la fiabilidad de la escala y de sus dimensiones se valoró calculando en índice de consistencia interna mediante el coeficiente Alpha de Cronbach, En la muestra estudiada se obtuvo una confiabilidad de .79 que puede ser valorado como indicador de una aceptable fiabilidad, ya que supera el punto de corte igual a .70 considerado para los instrumentos de medición (George y Mallery, 2003). Asimismo, se obtuvo la fiabilidad en las dimensiones. Para la validez del constructo se obtuvo los coeficientes de correlación ProductoMomento de Pearson ( $r$ ). Los coeficientes son altamente significativos, por ende, se confirma la validez de constructo de la escala. Igualmente, los coeficientes que resultan de la correlación entre ambas dimensiones son aceptables y significativos.

Escala de Resiliencia de Wagnild y Young.

La Escala de Resiliencia creada por Wagnild y Young (1993) mide la resiliencia y la clasifica en niveles. Consta de cinco factores, 1) satisfacción personal (4 ítems), 2) ecuanimidad (4 ítems), 3) sentirse bien solo (3 ítems), 4) confianza en sí mismo (7 ítems) y 5) perseverancia (7 ítems). En total consta de 25 ítems, que se responden en una escala tipo Likert de 7 puntos, donde 1 es totalmente en desacuerdo y 7 es máximo de totalmente de acuerdo, de este modo los participantes indican el grado de conformidad con el ítem; los puntajes altos son indicadores de niveles óptimos de resiliencia (Anexo 3). La prueba puede ser administrada de manera individual o colectiva. La escala total de la versión original de Wagnild y Young fue analizada por su confiabilidad y validez en Perú, fue adaptadatraducida al español por Novella (2002), quien, en una muestra de 324 personas de 14 a 17 años, utilizó la técnica de factorización y de rotación encuentra 2 factores, obtuvo con el coeficiente alfa una consistencia interna global de 0.875 , además correlaciones ítem-test que fluctuaban entre 0.18 a 0.63 , siendo todos los coeficientes estadísticamente significativos $(p<0.01)$.

Los puntajes directos se interpretan de la siguiente manera: bajo 1-131, moderado 132151 y alto 152-175. Además, los puntajes transformados a percentiles se interpretan de la siguiente manera: bajo de 1-25, moderado de 30-75 y alto de 80-99. Asimismo, para esta investigación la fiabilidad de la escala y de sus dimensiones se valoró calculando en índice de 
consistencia interna mediante el coeficiente Alpha de Cronbach. En la muestra estudiada la confiabilidad de la escala global (25 ítems) fue de .90 , que puede ser valorado como indicador de una excelente fiabilidad ya que supera el punto de corte igual a ,70 considerado para los instrumentos de medición (George y Mallery, 2003). Además, se puede apreciar que la fiabilidad de sus dimensiones son aceptables. Por otro lado, los coeficientes de correlación Producto-Momento de Pearson ( $r$ ) son altamente significativos, lo cual confirma que la escala presenta validez de constructo. Asimismo, los coeficientes que resultan de la correlación entre las dimensiones son aceptables y significativos.

\section{Análisis de datos}

La información recopilada fue procesada a través del software estadístico SPSS versión 23 aplicado para ciencias sociales. Los resultados fueron analizados, organizados y presentados en tablas descriptivas. Para contrastar la hipótesis planteada, dada la naturaleza de las variables, se aplicó la prueba estadística no paramétrica de Chi cuadrado (x2), puesto que, ambas variables no tienen un nivel de medición de tipo intervalo, de modo que tampoco se realizó la prueba de normalidad.

\section{RESULTADOS}

\section{Niveles de funcionamiento familiar}

En la tabla 1 se aprecia que la mayoría de los pacientes presenta un funcionamiento familiar medio (63.3\%), donde el tipo de familia que predomina es caóticamente conectada $(35.8 \%)$, es decir, en este tipo de familia existe considerable unión afectiva entre ellos, cierta lealtad, fidelidad e interdependencia entre los miembros con tendencia hacia la dependencia; sin embargo, también presentan ausencia de liderazgo, cambio aleatorio de roles, disciplina irregular y demasiados cambios. Por otro lado, se observa que existe un grupo considerable de pacientes con un funcionamiento familiar extremo $(22.9 \%)$, donde el tipo de familia que predomina es caóticamente amalgamada ( $20.2 \%)$, es decir, la familia que presenta ausencia de liderazgo, cambio aleatorio de roles, disciplina irregular y demasiados cambios, además, máxima unión efectiva entre los familiares, exigencia de fidelidad y lealtad a la familia, alto grado de dependencia para decisiones tomadas en común.

\begin{tabular}{lcc} 
& $n$ & $\%$ \\
Familias balanceadas & 15 & $13.8 \%$ \\
Flexiblemente separada & 6 & $5.5 \%$ \\
Flexiblemente conectada & 6 & $5.5 \%$ \\
Estructuralmente separada & 1 & $0.9 \%$ \\
Estructuralmente conectada & 2 & $1.8 \%$ \\
Familias medias & 69 & $63.3 \%$ \\
Flexiblemente desligada & 2 & $1.8 \%$ \\
Flexiblemente amalgamada & 2 & $1.8 \%$ \\
Caóticamente separada & 23 & $21.1 \%$ \\
Caóticamente conectada & 39 & $35.8 \%$ \\
Estructuralmente desligada & 1 & $0.9 \%$ \\
Estructuralmente amalgamada & 0 & $0.0 \%$ \\
Rígidamente separada & 1 & $0.9 \%$ \\
Rígidamente conectada & 1 & $0.9 \%$ \\
Familias extremas & 25 & $22.9 \%$ \\
Caóticamente desligada & 2 & $1.8 \%$ \\
Caóticamente amalgamada & 22 & $20.2 \%$ \\
Rígidamente desligada & 1 & $0.9 \%$ \\
Rígidamente amalgamada & 0 & $0.0 \%$ \\
\hline
\end{tabular}




\section{Niveles de resiliencia}

En la tabla 2 se observa que el $51.4 \%$ de los pacientes se ubican en un nivel moderado de resiliencia, lo cual sugiere que tienen moderada capacidad para disminuir o regular los efectos negativos del estrés, logrando soportar y sobrellevar las dificultades. Sin embargo, un grupo considerable de pacientes posee un nivel bajo de resiliencia. En cuanto a las dimensiones se observa que el $28.4 \%$ de los participantes se encuentra en un nivel alto de satisfacción personal, por ende, comprenden el significado de la vida y cómo se contribuye a esta.

\section{Tabla 2}

Nivel de resiliencia y sus dimensiones de los participantes.

\begin{tabular}{lcccccc} 
& \multicolumn{2}{c}{ Bajo } & \multicolumn{2}{c}{ Moderado } & \multicolumn{2}{c}{ Alto } \\
\cline { 2 - 7 } & $\mathrm{n}$ & $\%$ & $\mathrm{n}$ & $\%$ & $\mathrm{n}$ & $\%$ \\
Resiliencia & 28 & $25.7 \%$ & 56 & $51.4 \%$ & 25 & $22.9 \%$ \\
Satisfacción personal & 30 & $27.5 \%$ & 48 & $44.0 \%$ & 31 & $28.4 \%$ \\
Ecuanimidad & 28 & $25.7 \%$ & 54 & $49.5 \%$ & 27 & $24.8 \%$ \\
Sentirse bien solo & 30 & $27.5 \%$ & 50 & $45.9 \%$ & 29 & $26.6 \%$ \\
Perseverancia & 29 & $26.6 \%$ & 56 & $51.4 \%$ & 24 & $22.0 \%$ \\
Confianza en sí mismo & 29 & $26.6 \%$ & 53 & $48.6 \%$ & 27 & $24.8 \%$ \\
\hline
\end{tabular}

\section{Asociación entre funcionamiento familiar y resiliencia}

En la tabla 3 se aprecia que no existe asociación significativa entre el nivel de funcionamiento familiar y el nivel de resiliencia $(x 2=5,972, p>.05)$. De igual manera, no se observa relación significativa en las dimensiones de resiliencia y funcionamiento familiar.

\section{Tabla 3}

Asociación entre funcionamiento familiar y resiliencia en los participantes.

\begin{tabular}{lccc} 
& \multicolumn{2}{c}{ Funcionamiento familiar } & \\
& $\mathrm{x} 2$ & $\mathrm{~g}$ & $\mathrm{p}$ \\
Resiliencia & 5.972 & 4 & .201 \\
Satisfacción & 4.757 & 4 & .270 \\
Ecuanimidad & 7.054 & 4 & .133 \\
Sentirse & 3.339 & 4 & .503 \\
Perseverancia & 2.931 & 4 & .569 \\
Confianza en sí mismo & 8.441 & 4 & .077 \\
\hline
\end{tabular}

\section{DISCUSIÓN}

A continuación, se lleva a cabo el análisis de los resultados de asociación entre funcionamiento familiar y resiliencia en pacientes con enfermedades no transmisibles.
En esta investigación se halló que el funcionamiento familiar no se asocia con la resiliencia ( $x 2=5,972 ; p>.05)$. Asimismo, Castro y Morales (2014) presentaron resultados similares, donde no se encontró relación entre clima social familiar y resiliencia, puesto que, la dinámica de estas variables podría estar relacionada a otros factores como el centro 
educativo, la tecnología, cultura y comunidad. La resiliencia desde el modelo de Wagnild y Young estaría como una característica de la personalidad, la cual regula el efecto negativo del evento estresor y promueve su adaptación, por ende, la capacidad de resistir, tolerar la presión, enfrentar obstáculos y estar firme en sus convicciones, está presente cualquiera sea la circunstancia. En consecuencia, el adolescente debe construir conscientemente su propia resiliencia y desarrollar estrategias resilientes para superar las adversidades, diseñando respuestas positivas y estrategias para resolver problemas (Rodríguez, 2009).

Además, se encontró que no existe asociación entre funcionamiento familiar y la dimensión satisfacción personal ( $x 2=4.757 ; p>.05)$. Siendo congruente con el modelo circumplejo de Olson, el funcionamiento familiar es considerado como la capacidad de afronte que la familia posee ante los problemas, sin embargo, satisfacción personal es una dimensión intrínseca del ser humano, mostrando que posee comprensión hacia el significado de la vida. Martínez-Antón, Buelga y Cava (2007) encontraron relación entre variables escolares (ajuste social y clima social) e indicadores de ajuste psicosocial (autoestima, victimización y satisfacción con la vida), ambas variables estaban indirectamente relacionadas debido a la presencia de satisfacción con la vida, además, tuvo relación positiva significativa con autoestima, sin embargo, con victimización obtuvo relación negativa significativa. Asimismo, las condicionantes internas tienen mayor énfasis a las condiciones externas, por ende, la autovaloración y la jerarquía motivacional podrían estar más relacionadas que el funcionamiento familiar o el grupo de pares, puesto que depende del individuo cuan importantes son los valores sociales para el nivel de satisfacción personal alcanzado (GarcíaViniegras y Rodríguez, 2007). En este sentido, se puede decir que satisfacción personal estaría ligada más a factores intrínsecos.

Del mismo modo, se encontró que el funcionamiento familiar no se asocia con la ecuanimidad (x2=7.054; $p>.05)$. Ayudan a explicar este hallazgo, los resultados obtenidos por Torres (2006), quien encontró relación entre características personales y sentido del humor, lo cual es significativo para nuestra investigación porque la presencia de ecuanimidad, energía, entusiasmo y alegría (que en el trabajo de Torres se asocian al buen humor) son consideradas como características personales. Entonces la interpretación y aprendizaje que obtiene el paciente por medio de sus experiencias en el proceso de llevar la enfermedad parte de una cualidad personal, a pesar del entorno que lo rodea. Para Wagnild y Young la ecuanimidad es la perspectiva balanceada que la persona tiene de su vida y experiencias, que le permite reaccionar racionalmente y con actitudes positivas ante la adversidad. Además, Peter Rothschild (Citado por Senado, 1999) menciona que ante la enfermedad nadie es capaz de observar con ecuanimidad la decadencia de su cuerpo, sin embargo, Guil et al. (2016) encontraron niveles altos de ecuanimidad relacionados a bienestar psicológico y las dimensiones de optimismo (auto-aceptación y propósito en la vida) en mujeres con cáncer de mama. Se podría afirmar que la ecuanimidad es una cualidad intrínseca e innata de la persona.

Asimismo, no se halló asociación entre funcionamiento familiar y sentirse bien solo $(x 2=3.339 ; p>.05)$. En una investigación se encontró que las dimensiones sentirse bien solo y perseverancia de resiliencia tienen una relación negativa con el consumo de tabaco, alcohol y drogas, por el contrario, tendría una relación positiva con conductas de salud (Antonio et al, 2015). Además, Quintero, Jabela, Rocha y Serna (2015) encontraron que los adolescentes prefieren trabajar solos, que la compañía de sus familiares, amigos o el diálogo continuo para el conjunto de sus motivaciones intrínsecas no es un factor indispensable para el desarrollo de su resiliencia.

Del mismo modo, no fue hallada asociación entre funcionamiento familiar y confianza en sí mismo $(x 2=8.441 ; p>.05)$. Los resultados obtenidos por Calle (2017) mostraron relación significativa entre inteligencia emocional y confianza en sí mismo en pacientes con cáncer gástrico. Es decir, aquellos que poseían más habilidades para conocer y regular sus emociones, así como para percibir las emociones de otros, tenían mayor orgullo de sus logros, confianza para enfrentar dificultades y para apoyar a otros en tiempos difíciles. Por otro lado, Vázquez y Pérez-Sales (2003) mencionaron que es significativo destacar que cuando las personas atraviesan por eventos traumáticos, tienden a mostrar ciertas capacidades y destrezas que hacen que aumente la confianza en sí mismos y por ende puedan tener mayor capacidad para enfrentar nuevos eventos negativos. Entonces, 
es posible que la confianza en sí mismo no se relacione obligatoriamente a factores externos como la familia o amigos, sino de habilidades personales, las cuales son reforzadas por la experiencia. Este razonamiento concuerda con el de Wagnild y Young (1993) quienes sostienen que la confianza en sí mismo es una habilidad que la persona posee, gracias a la cual confía en sus capacidades.

Por último, no se encontró asociación entre funcionamiento familiar y perseverancia ( $x 2$ $=2.931 ; \mathrm{p}>0.05)$. Podríamos considerar la perseverancia como una característica propia del ser, la cual se desarrolla en un medio poco favorable, que es alimentada por la experiencia obtenida por previos eventos fortuitos y por la motivación intrínseca. Esta definición se afirma con la investigación de Werner (1993) la cual tuvo como resultado que los niños que experimentan temor y culpa ante la existencia de violencia activa y pasiva en sus hogares, se desempeñaban de manera satisfactoria en sus actividades escolares o del hogar, por lo tanto, desarrollaron la capacidad de perseverancia y solución de problemas. Asimismo, Becoña (2006) plantea que la persona resiliente ante adversidades o en un sistema familiar que no permite su desarrollo, sale victoriosa, pues toma las dificultades como una oportunidad y persevera hasta salir airoso del problema.

\section{Declaración de financiamiento y de conflictos de interés:}

El estudio fue financiado por los autores, quienes declaran no tener conflictos de interés.

\section{Correspondencia}

Moises Isaac Cespedes Felix

Correo electrónico: moisescespedes@upeu. edu.pe

Jesly Alexandra Nina Cayte

Correo electrónico: jeslynina@upeu.edu.pe

\section{REFERENCIAS BIBLIOGRÁFICAS}

Antonio, M., Arrioja, G., De León, A., Velasco, L., Torres, A., Adrían, F., Coyolt, E., Xicali, N., Tenahua, I, Villegas, M. \& Torres, M. (2015). Resiliencia, conducta de salud y conducta de consumo de drogas en estudiantes universitarios. European Journal of Child Development, 3(2), 55-67. doi: 10.1989/ejpad.v3i2.29

Athié, D., \& Gallegos, P. (2009). Relación entre la resiliencia y el funcionamiento familiar. Psicología Iberoamericana, 17(1), 5-14. Recuperado de: http://www.redalyc.org/pdf/1339/133912613002. pdf

Becoña, E. (2006). Resiliencia: definición, características y utilidad del concepto. Revista de Psicopatología y Psicología Clínica,11(3), 125-146.Recuperado de: http://aepcp.net/ arc/01.2006(3).Becona.pdf

Bonanno, G. (2004). Loss, trauma and human resilience: Have we underestimated the human capacity to thrive after extremely aversive events? American Psychologist, 59(1), 20-28. Recuperado de: https://www.tc.columbia.edu/faculty/gab38/ faculty-profile/files/americanPsychologist.pdf

Calle, E. (2017). Inteligencia emocional y resiliencia en pacientes con cáncer gástrico y sin antecedentes de violencia familiar de un hospital de Lima, 2017 (Tesis de licenciatura publicada). Universidad César Vallejo, Lima. Recuperado de: http:// repositorio.ucv.edu.pe/handle/UCV/8501

Castro, G. \& Morales, A. (2014). Clima social familiar y resiliencia en adolescentes de cuarto año de secundaria de una institución educativa estatal en Chiclayo, 2013. (Tesis de licenciatura publicada). Universidad Católica Santo Toribio de Mogrovejo, Chiclayo. Recuperado de: http:// tesis.usat.edu.pe/bitstream/usat/348/1/TL CastroCruzadoGlory_MoralesRoncalAngelica. pdf

Florenzano, R. (1995). Familia y salud de los jóvenes. Chile: Edición Universidad Católica de Chile. Recuperado de: http://www.ts.ucr.ac.cr/binarios/ congresos/reg/slets/slets-017-033.pdf

Florenzano, R. (1998). El adolescente y sus conductas de riesgo. Chile: Ed. Universidad Católica de Chile.

García-Viniegras, C. \& Rodríguez, G. (2007). Calidad de vida en enfermos crónicos. Revista Habanera de Ciencias Médicas, 6(4). Recuperado de: http://scielo.sld.cu/scielo.php?script=sci_ arttext\&pid=S1729-519X2007000400002

George, D. \& Mallery, P. (2003). SPSS for Windows step by step: A simple guide and reference. 
Boston: Editorial Allyn \& Bacon. Guil, R., Zayas, A., Gil-Olarte, P., Guerrero, C., Gonzáles, S. \& Mestre, M. (2016). Bienestar psicológico, optimismo y resiliencia en mujeres con cáncer de mama. Psicooncología, 13(1), 127-138. Doi: 10.5209/rev_PSIC.2016.v13.n1.52492

Hernández, R., Fernández, C., \& Baptista, P. (2005). Metodología de la Investigación. México: McGraw-Hill Interamericana.

Juárez, A. (2012). Construir desde las fortalezas: trabajo social y resiliencia. Revista de ciencias humanas y sociales. 136(70), 5-36.Recuperado de: http://revistas.upcomillas.es/index.php/ miscelaneacomillas/article/view/718/594

Ledón, L. (2011). Enfermedades crónicas y vida cotidiana. Revista Cubana de Salud Pública, 37(4), 488-499. Recuperado de: http://scielo.sld.cu/scielo.php?script=sci_ arttext\&pid=S0864-34662011000400013

Leyva-Jiménez, R., Hernández-Juárez, A., NavaJiménez, G., \& López-Gaona, V. (2007). Depresión en adolescentes y funcionamiento familiar. Revista Médica del Instituto Mexicano del Seguro Social, 45(3), 225-232.

Martínez-Antón, M., Buelga, S. \& Cava, J. (2007). La satisfacción con la vida en la adolescencia y su relación con la autoestima y el ajuste escolar. Anuario de Psicología Universitat de Barcelona, 38(2), 293-303. Recuperado de: http://www. raco.cat/index.php/anuariopsicologia/article/ viewFile/74205/94371

Novella, A. (2002). Incremento de la resiliencia luego de la aplicación de un programa de psicoterapia breve en madres adolescentes (Tesis para optar el grado académico de Magíster en Psicología). Universidad Nacional Mayor de San Marcos.

Olson, D. (1979). El funcionamiento familiar en el modelo circumplejo de Olson, Russell y Sprenkle. En Aquilino, P. L., \& Martinez, P. (Eds), Evaluación Psicológica y psicopatológica de la familia (pp. 195-137). Madrid: Ediciones Rialp.

Olson, D., Portner, J. \& Lavee, Y. (1985). FACES III: Family adaptability and cohesion evaluation scales. New York: Oxfor University Press.

Organización Mundial de la Salud (2017). Enfermedades No Transmisibles. Recuperado de: http://www.who.int/mediacentre/factsheets/ fs $355 /$ es/

Organización Mundial de la Salud (2017). Enfermedades crónicas. Recuperado de http:// www.who.int/topics/chronic_diseases/es/ Quintero, A., Jabela, J., Rocha, M. \& Serna, A. (2015). La resiliencia en adolescents de 13 a 15 años y jóvenes de 16 a 18 años privados de la libertad del centro de reclusion Luis A. Rengifo en la ciudad de lbagué. Revista de investigaciones de la Escuelna de Ciencias Sociales, Artes y Humanidades, 6, 37-45. Recuperado de: http:// hemeroteca.unad.edu.co/index.php/desbordes/ article/view/1863/2078

Reusche, R. (1994). El modelo circumplejo de funcionamiento familiar en la estructura familiar de adolescentes de nivel socio-económico medio: un estudio exploratorio en residentes en Lima. (Tesis de maestría publicada). Universidad Femenina del Sagrado Corazón. Recuperado de: http://www.unife.edu.pe/publicaciones/revistas/ psicologia/2013/2_quezada.pdf

Rodríguez, A. (2009). Resiliencia. Revista Psicopedagógica, 26(80), 291302. Recuperado de: http://pepsic. bvsalud.org/scielo.php? script = sci_ arttext\&pid=S0103-84862009000200014

Senado, J. (1999). Los factores de riesgo en el proceso salud-enfermedad. Revista cubana de medicina general integral, 15(4), 453-460. Recuperado de: http://scielo.sld.cu/pdf/mgi/v15n4/mgi19499.pdf

Torres, H. (2006). Características personales del sentido del humor en áreas laborales. Revista de Estudios Interdisciplinarios en Ciencias Sociales, 8(1), 122-128. Recuperado de: http://www. redalyc.org/pdf/993/99318655009.pdf

Vázquez, C., \& Pérez-Sales, P. (2003). Emociones positivas, trauma y resistencia. Ansiedad y Estrés, 9(3), 231-254. Recuperado de: http:// www.pauperez.cat/en/thematic/articles/identityand-beliefs/19-emociones-positivas-trauma-yresistencia-ansiedad-y-estres/file

Wagnild G. y Young H. (1993). Development and Psychometric Evaluation of the Resilience Scale. Journal of Nursing Measurement, 1(2). Recuperation of: https://www. academia.edu/6675361/Development_and_ Psychometric_Evaluation_of_the_Resilience_ Scale?auto=download

Werner, E. (1993). Protective factors and individual resilience. En Meisels, S. y Shonkof, J. (Eds.), Handbook of early childhood intervention (pp.115). Nueva York: Cambridge University Press. Recuperation of: http://catdir.loc.gov/ catdir/samples/cam032/99025362.pdf

Recibido: 04/03/18 Aceptado: 09/07/18 